\title{
Phospho-S6 ribosomal protein: a potential new predictive sarcoma marker for targeted mTOR therapy
}

\author{
O Hans Iwenofu ${ }^{1,2}$, Richard D Lackman ${ }^{3}$, Arthur P Staddon ${ }^{4}$, Diana G Goodwin ${ }^{1}$, \\ Helen M Haupt ${ }^{1}$ and John SJ Brooks ${ }^{1}$
}

\begin{abstract}
${ }^{1}$ Department of Pathology \& Laboratory Medicine, Pennsylvania Hospital of the University of Pennsylvania Health System, Philadelphia, PA, USA; ${ }^{2}$ Department of Pathology \& Laboratory Medicine, Ohio State University, Columbus, OH, USA; ${ }^{3}$ Department of Orthopaedic Surgery, Pennsylvania Hospital of the University of Pennsylvania Health System, Philadelphia, PA, USA and ${ }^{4}$ Department of Hematology $\&$ Oncology, Pennsylvania Hospital, Philadelphia, PA, USA
\end{abstract}

\begin{abstract}
Metastatic sarcomas are commonly resistant to chemotherapy. The serine/threonine kinase, mammalian target of rapamycin (mTOR), is a protein kinase of the phosphatidylinositol 3-kinase (PI3K)/AKT signaling pathway thought to have a key role in controlling cancer growth and thus is an important target for cancer therapy. Several inhibitors of mTOR are in clinical trials, including AP23573, which is being tested on metastatic sarcomas and other tumors. We hypothesized that a marker for the activity of mTOR, phosphorylated S6 ribosomal protein, would be predictive of clinical response to the drug, that is, high tumor expression would signify better response than low expression. This was a blinded study. Of 26 patients treated, 20 remained on study, with available paraffin blocks. Fourteen patients received AP23573 alone and six patients received AP23573 in combination with adriamycin. An antibody to the phosphorylated S6 ribosomal protein was used to stain the tumors, all high-grade sarcomas. Pretreatment biopsy or resection material was tested: the original tumor $(n=6)$ or tumor recurrence/metastasis $(n=14)$; either of these may have been after treatment with other agents. Staining was scored for both quantity/percentage of tumor cells and intensity. Scoring was performed without knowledge of tumor response. Staining quantity could be categorized into two natural groups: high expressors ( $\geq 20 \%$ of tumor cells, 11 cases) and low expressors (0-10\% of tumor cells, 9 cases). The highexpression group had eight stable and three progressive cases (73\% stable disease); the low-expression group had three stable and six progressive cases (67\% progressive disease). Chi-square analysis showed statistical significance $(P \leq 0.05)$ at this initial cutoff $(10 \%)$ selected blindly. The level of phosphorylated S6 ribosomal protein expression was predictive of early tumor response to the $\mathrm{mTOR}$ inhibitor, suggesting that this is a promising new predictive sarcoma marker for targeted mTOR inhibitor therapy.

Modern Pathology (2008) 21, 231-237; doi:10.1038/modpathol.3800995; published online 21 December 2007
\end{abstract}

Keywords: phospho-S6 ribosomal protein; sarcoma marker; mTOR inhibitor; sarcoma therapy; predictive marker; immunohistochemistry

Soft tissue sarcomas are rare malignancies of mesenchymal origin. While there have been significant strides in the past 30 years in the management

Correspondence: Dr JSJ Brooks, MD, FRCPath, Department of Pathology, Pennsylvania Hospital of the University of Pennsylvania Health System, 800 Spruce Street, Philadelphia, PA 19107, USA.

E-mail: john.brooks@uphs.upenn.edu

This work was presented in part at the 95th annual meeting of the United States and Canadian Academy of Pathology, March 24-30, 2007, San Diego, CA, USA; it received a Stowell-Orbison Award Certificate of Merit.

Received 23 July 2007; revised 21 September 2007; accepted 01 October 2007; published online 21 December 2007 of these neoplasms with better chemotherapy regimens, ${ }^{1}$ the treatment outcomes are still far from optimal. The drawbacks of the conventional multimodality approach of surgery, with or without chemoradiation, include significant morbidity from the debilitative effects of surgery and systemic toxicity from chemotherapy. Furthermore, metastatic sarcomas are commonly resistant to this conventional treatment approach.

Recent progress in the understanding of the biology of these rare tumors has enabled the identification of distinct molecular and pathologic entities within this heterogenous group of tumors and has paved the way for the development of 
targeted therapy against activated kinases. ${ }^{2,3}$ One example is the identification of KIT and platelet-derived growth factor receptor-a (PDGFRA) and platelet-derived growth factor receptor-b (PDGFRB) kinase mutations in gastrointestinal stromal tumors and dermatofibrosarcoma protuberans, respectively, which led to the development of imatinib, sunitinib and other tyrosine kinase inhibitors for the treatment of solid tumors. ${ }^{4,5}$

Mammalian target of rapamycin (mTOR) is a ubiquitously expressed and highly conserved serine/threonine kinase that affects a number of cellular functions, including protein synthesis and cell proliferation, and also plays a key regulatory role in cell signaling pathways that respond to both growth factors and nutritional status. ${ }^{6-11}$ mTOR is a key component of the phosphatidylinositol 3-kinase (PI3K) and protein kinase B (AKT) pathways that activate downstream kinases required for $\mathrm{G} 1$ to $\mathrm{S}$ phase transition. ${ }^{12}$ mTOR acts by directly activating p70S6 kinase (p70S6K/S6K1) and inhibiting 4E binding protein 1 (4E-BP1) ${ }^{6-9}$ p70S6K is a serine/ threonine kinase that phosphorylates the S6 protein of the 40S ribosomal subunit (phosphorylated S6 ribosomal protein (phospho-S6rp)) at several sites, including serines 235 and 236, leading to initiation of protein synthesis. ${ }^{13,14} 4 \mathrm{E}-\mathrm{BP} 1$ is a translational repressor that negatively regulates eukaryotic initiation factor $4 \mathrm{E} / 4 \mathrm{G}$ (eIF-4E/G) complex by modulating phosphorylation of the involved proteins ${ }^{15}$ (Figure 1). The exact mechanisms leading to mTOR activation is unclear, but certain nutrients and growth factors are believed to act as indirect activators of mTOR. ${ }^{16,17}$

There are growing data that indicate that mTOR is frequently activated in many cancers as a result of alterations in the PI3K/AKT pathway and thus presents a potential therapeutic target. ${ }^{9,12,18}$ Several inhibitors of mTOR are in clinical trials; AP23573 is one of them and has demonstrated potent antitumor activity in non-clinical and early clinical studies. $^{9,12,18,19}$

The objectives of this study were two-fold: (1) to determine whether there was phospho-S6rp immunoreactivity in sarcomas and whether this reactivity was variable among sarcoma types; and (2) to ascertain in a blinded manner if there was any correlation between the level of phospho-S6rp expression by immunohistochemistry and early clinical response to AP23573. Accordingly, we formulated a hypothesis that a marker for the activity of the mTOR pathway (phospho-S6rp)

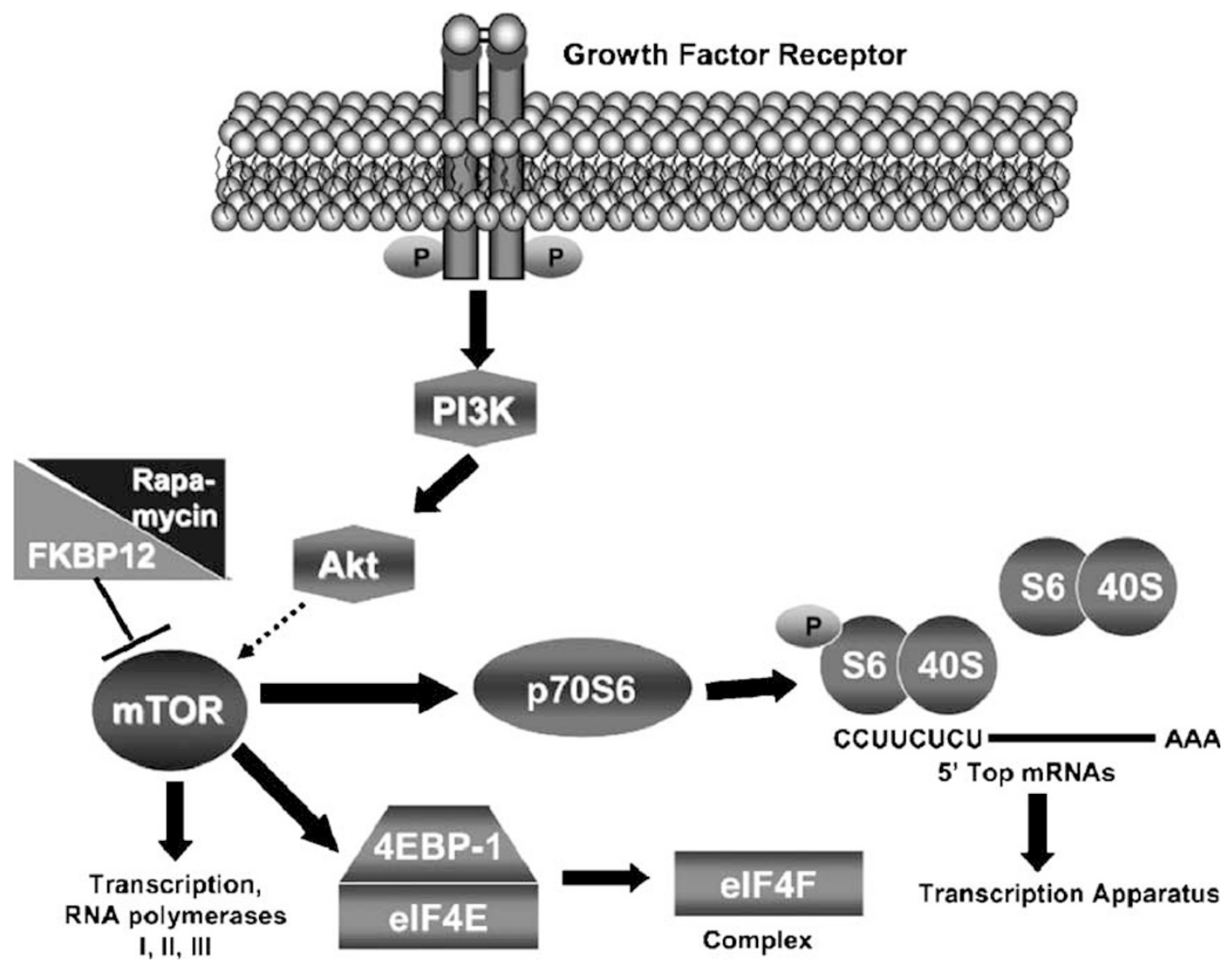

Figure 1 Schematic of PI3K/AKT/mTOR pathway (from Rowinsky EK. Targeting the molecular target of rapamycin (mTOR). Curr Opin Oncol 2004;16:564-575). 
would be predictive for the clinical response to the mTOR inhibitor AP23573, that is, high tumor expression would signify better response than low expression.

\section{Materials and methods}

\section{Study Design/Patient Selection}

This was a blinded analysis of specimens from patients undergoing treatment at the Pennsylvania Hospital as part of two multicenter trials of AP23573. Of 26 patients treated at our institution, tissue blocks were available on 20 patients. Fourteen patients were enrolled in a single-agent study in which AP23573 was administered by intravenous injection for 5 consecutive days every other week. Six patients were enrolled in a combination study in which AP23573 was administered orally on one of two schedules (daily for 4 days followed by 3 days of rest or daily for 14 days followed by 7 days of rest) in combination with doxorubicin (every 3 weeks). For the purpose of this analysis, the results of treatment were evaluated at 2 months as stable (S) or progressive $(\mathrm{P})$ disease based on RECIST criteria $^{20}$ (one patient evaluated as stable had a partial response (case no. 4)). All 20 patients treated in both studies at the Pennsylvania Hospital who had paraffin blocks available and were evaluable for clinical response at 2 months were included in this analysis. Treatment results were unknown to the pathologists at the time of scoring the immuno- histochemical staining and were correlated with immunohistochemical results after scoring was performed.

Pretreatment biopsy or resection material was tested; wherever possible, the material most immediately before treatment with AP23573 was used for staining. The original slides of either the pretreatment biopsy or/and the resection specimen were retrieved and reviewed by two pathologists to validate the diagnosis. With the exception of osteosarcomas, tumors were diagnosed and graded according to the FNCLCC (French Federation Nationale des Centres de Lutte Contre le Cancer) system. ${ }^{21}$ Osteosarcomas were all osteoblastic grade 3 of 3 (high grade). Cases included the original (ie, primary) tumor $(n=6)$ or tumor recurrence/ metastasis $(n=14)$ (Table 1). Tumors included pleomorphic undifferentiated sarcoma ( $\mathrm{MFH}, n=5)$, osteosarcoma $(n=3)$, leiomyosarcoma $(n=5)$, liposarcoma $(n=3)$ and one each of malignant peripheral nerve sheath tumor (MPNST), synovial sarcoma, malignant solitary fibrous tumor and sarcoma NOS.

\section{Immunohistochemistry}

Formalin-fixed, paraffin-embedded tissue was cut at $3 \mu \mathrm{m}$, mounted on positively charged slides and dried at $58^{\circ} \mathrm{C}$ for $30 \mathrm{~min}$. Immunohistochemical staining was performed on the Ventana Benchmark ${ }^{\circledR}$ XT Automated IHC Stainer using the Ventana iView $^{\mathrm{TM}}$ DAB detection kit (catalog no. 760-091).

Table 1 Clinical summary

\begin{tabular}{|c|c|c|c|c|}
\hline \multirow[t]{2}{*}{ Case no. } & \multirow{2}{*}{$\begin{array}{c}\text { Age } \\
\text { (years)/sex }(M / F)\end{array}$} & \multicolumn{2}{|r|}{ Site } & \multirow[t]{2}{*}{ Diagnosis } \\
\hline & & Primary & Relapse tissue & \\
\hline 1 & $50 \mathrm{~F}$ & Left proximal tibia & Lung (met.) ${ }^{\mathrm{a}}$ & Pleomorphic undiff. sarcoma, grade 3 \\
\hline 2 & $18 \mathrm{M}$ & Right distal femur & Right distal femur (rec.) $^{\mathrm{a}}$ & Osteogenic sarcoma, osteoblastic type, grade $3 / 3$ \\
\hline 3 & $36 \mathrm{~F}$ & Paraspinal & Paraspinal (rec.) ${ }^{\mathrm{a}}$ & Pleomorphic undiff. sarcoma, grade 3 \\
\hline 4 & $36 \mathrm{~F}$ & Right shoulder & Left paraspinal (met.) ${ }^{\mathrm{a}}$ & Pleomorphic undiff. sarcoma, grade 3 \\
\hline 5 & $75 \mathrm{M}$ & Right calf & Lung (met) ${ }^{\mathrm{a}}$ & Leiomyosarcoma, grade 2 \\
\hline 6 & $49 \mathrm{M}$ & Right groin & $\begin{array}{l}\text { Left lower lung lobe (met.) } \\
\text { Left upper lung lobe (met.) }\end{array}$ & Synovial sarcoma, monophasic, grade 3 \\
\hline $7^{\mathrm{b}}$ & $28 \mathrm{M}$ & Right pelvis ${ }^{\mathrm{a}}$ & None & Malignant peripheral nerve sheath tumor, grade 3 \\
\hline $8^{\mathrm{b}}$ & $40 \mathrm{M}$ & Left shoulder & Left shoulder (rec.) ${ }^{\mathrm{a}}$ & Malignant solitary fibrous tumor, grade 3 \\
\hline $9^{\mathrm{b}}$ & $36 \mathrm{M}$ & Intra-abdominal $^{\mathrm{a}}$ & None & Liposarcoma, myxoid/round cell, grade 2 \\
\hline 10 & $47 \mathrm{~F}$ & Small intestine/colon & Small intestine/colon (rec.) ${ }^{\mathrm{a}}$ & Pleomorphic undiff. sarcoma, grade 3 \\
\hline 11 & $51 \mathrm{~F}$ & Left popliteal fossa & Lung (met.) ${ }^{\mathrm{a}}$ & Pleomorphic undiff. sarcoma, grade 3 \\
\hline 12 & $48 \mathrm{~F}$ & Stomach $^{\mathrm{a}}$ & None & Leiomyosarcoma, grade 2 \\
\hline 13 & $48 \mathrm{M}$ & Right leg & Pelvis (met.) ${ }^{\mathrm{a}}$ & Liposarcoma, myxoid, grade 2 \\
\hline 14 & $29 \mathrm{M}$ & Left distal femur & Right lower lung lobe (met.) ${ }^{a}$ & Osteogenic sarcoma, osteoblastic type, grade $3 / 3$ \\
\hline 15 & $69 \mathrm{~F}$ & Kidney & Multiple lung mets. $^{\mathrm{a}}$ & Leiomyosarcoma, grade 3 \\
\hline 16 & $60 \mathrm{~F}$ & Unknown & Right lower lung lobe (met.) ${ }^{a}$ & Leiomyosarcoma, grade 2 \\
\hline $17^{\mathrm{b}}$ & $43 \mathrm{~F}$ & Paraspinal & Paraspinal (rec.) ${ }^{\mathrm{a}}$ & Sarcoma, NOS, grade 3 \\
\hline $18^{\mathrm{b}}$ & $64 \mathrm{M}$ & Retroperitoneal $^{\mathrm{a}}$ & None & Leiomyosarcoma, grade 2 \\
\hline $19^{\mathrm{b}}$ & $54 \mathrm{M}$ & Right pelvis ${ }^{\mathrm{a}}$ & None & Liposarcoma, pleomorphic, grade 3 \\
\hline 20 & $17 \mathrm{M}$ & Right $\operatorname{arm}^{\mathrm{a}}$ & None & Osteogenic sarcoma, osteoblastic type, grade $3 / 3$ \\
\hline
\end{tabular}

Undiff. $=$ undifferentiated; met. $=$ metastasis; rec. = local recurrence

${ }^{\mathrm{a}}$ Specimen tested for phospho-S6rp.

b Patients who received AP23573 in combination with doxorubicin. 
Each step of the iView DAB detection kit procedure was optimized on the Benchmark XT and was preset. Ventana High Temperature Liquid Coverslip (catalog no. 650-010) was used throughout the automated protocol as appropriate. Likewise, the slides were rinsed between steps with Ventana Tris-based Reaction Buffer (catalog no. 950-300).

Following deparaffinization with Ventana EZ Prep solution (catalog no. 950-102), antigen retrieval was performed using Ventana proprietary Tris-based buffer solution CC1 (catalog no. 950-124) at $95-100^{\circ} \mathrm{C}$ for $30 \mathrm{~min}$. Endogenous peroxidase was blocked with iView inhibitor, $3 \% \mathrm{H}_{2} \mathrm{O}_{2}$, for 4 min at $37^{\circ} \mathrm{C}$. After rinsing, the slides were incubated at $37^{\circ} \mathrm{C}$ for $32 \mathrm{~min}$ with a 1:50 dilution of phosphoS6rp (Ser235/236) (91B2) rabbit monoclonal concentrated primary antibody (catalog no. 4857; Cell Signaling Technology). Signal enhancement was performed using the Ventana Amplification kit (catalog no. 760-080) for two incubations of $8 \mathrm{~min}$ each. Slides were then incubated with iView biotinylated goat anti-rabbit IgG and IgM secondary antibody, followed by iView streptavidin horseradish peroxidase conjugate, each for $8 \mathrm{~min}$. Visualization was achieved using iView DAB $(2 \mathrm{~g} / \mathrm{l})$ substrate with $0.04 \% \mathrm{H}_{2} \mathrm{O}_{2}$ added for $8 \mathrm{~min}$, followed by enhancement with $\mathrm{CuSO}_{4}(5 \mathrm{~g} / \mathrm{l})$ for 4 min. Slides were then counterstained for $4 \mathrm{~min}$ with hematoxylin and rinsed. After removing from the instrument, slides were manually dehydrated and coverslipped. For a positive control, a colon carcinoma was used, since these are known to have mTOR activation at high frequency. ${ }^{9}$ This positive control was run with each tumor tested and exhibited strong staining in the carcinoma. For a negative antibody control, tumors were tested without the primary phospho-S6rp antibody with each run. Negative tissue controls were contained within the colon carcinoma control (lack of staining in resting fibroblasts, normal vascular smooth muscle and normal muscularis propria (which occasionally showed trace staining)).

\section{Quantitation of Immunohistochemistry}

Staining was scored for both quantity of tumor cells (\%: $0 \%, \leq 10 \%, \leq 25 \%, \leq 50 \%,>50 \%$ ) and intensity (0, negative; $1+$, weak staining; $2+$, intermediate staining; $3+$, strong staining). Scoring was performed without knowledge of tumor response.

\section{Results}

All of the tumors were high grade (grade 2 or 3 ) and included pleomorphic undifferentiated sarcoma (malignant fibrous histiocytoma (5), osteogenic sarcoma (3), leiomyosarcoma (5), liposarcoma (3) and one each of synovial sarcoma, MPNST, malignant solitary fibrous tumor and sarcoma NOS) (Table 1).
Table 2 phospho-S6rp immunohistochemical characteristics

\begin{tabular}{|c|c|c|c|c|c|c|c|}
\hline \multicolumn{4}{|c|}{ Low expressors } & \multicolumn{4}{|c|}{ High expressors } \\
\hline $\begin{array}{l}\text { Case } \\
\text { no. }\end{array}$ & $\begin{array}{c}\% \\
\text { Staining }\end{array}$ & Intensity & $S / P$ & $\begin{array}{c}\text { Case } \\
\text { no. }\end{array}$ & $\begin{array}{c}\% \\
\text { Staining }\end{array}$ & Intensity & $S / P$ \\
\hline 1 & 5 & $2+$ & S & 4 & 40 & $2+$ & S \\
\hline 2 & 0 & 0 & $S$ & 5 & 75 & $1+$ & S \\
\hline 3 & 5 & $2+$ & $\mathrm{S}$ & 7 & 30 & $2+$ & S \\
\hline 6 & 0 & 0 & $\mathrm{P}$ & 9 & 50 & $3+$ & $\mathrm{P}$ \\
\hline 8 & 0 & 0 & $\mathrm{P}$ & 10 & 40 & $3+$ & S \\
\hline 14 & 10 & $2+$ & $\mathrm{P}$ & 11 & 70 & $1+$ & S \\
\hline 15 & 5 & $2+$ & $\mathrm{P}$ & 12 & 30 & $2+$ & S \\
\hline 17 & 0 & 0 & $\mathrm{P}$ & 13 & 20 & $3+$ & $\mathrm{P}$ \\
\hline \multirow[t]{3}{*}{20} & 0 & 0 & $\mathrm{P}$ & 16 & 95 & $3+$ & S \\
\hline & & & & 18 & 25 & $3+$ & $\mathrm{P}$ \\
\hline & & & & 19 & 90 & $3+$ & S \\
\hline
\end{tabular}

$\mathrm{S}=$ stable $\mathrm{P}=$ progressive

Table 3 Chi-square analysis of phospho-S6rp expression vs clinical response

\begin{tabular}{lcc}
\hline & Low expressors & High expressors \\
\hline Stable disease (S) & 3 & 8 \\
Progressive disease (P) & 6 & 3 \\
\hline
\end{tabular}

It was immediately apparent that the immunohistochemical staining pattern fell into two natural groups: low expressors (LE, $0-10 \%$ of tumor cells, 9 cases) and high expressors (HE, $\geq 20 \%$ of tumor cells, 11 cases; Table 2). Strong intensity $(2+/ 3+)$ was present in 13 of the 15 positive cases. In the HE group, 9 of 11 positive cases had strong expression. No staining was seen in 5 of the 20 cases.

In the HE group, there were eight stable (S) and three progressive (P) cases $(73 \% \mathrm{~S})$ after two cycles of treatment with AP23573 or AP23573 and doxorubicin; in the LE group, there were three $\mathrm{S}$ and six $\mathrm{P}$ cases $(67 \% \mathrm{P})$. Interestingly, four of the five negative cases had progressive disease. Chi-square analysis had statistical significance $(P \leq 0.05)$ at this blindly selected cutoff of $10 \%$ (Table 3 ). Furthermore, two of the three progressors in the HE group had the lowest quantity of scores (20 and 25\%). Even if only the 14 recurrent tumors are analyzed, the percentages remain the same: high expression in six cases with five having stable disease $(5 / 6,84 \%$ stable), and low expression in eight cases with five progressive cases $(5 / 8,62 \%$ progressive disease). In this limited cohort, we also noticed highly variable staining patterns within and between the sarcoma subsets; for example, osteogenic sarcoma (0-2+, Figure 2), leiomyosarcoma (1-3+, Figure 3) and malignant fibrous histiocytoma $(1-3+)$. In liposarcoma, while all three cases had strong staining intensity, $(3+)$ there was significant variation in staining quantity (20-90\%, Figure 4). No staining was observed in our cases of synovial 

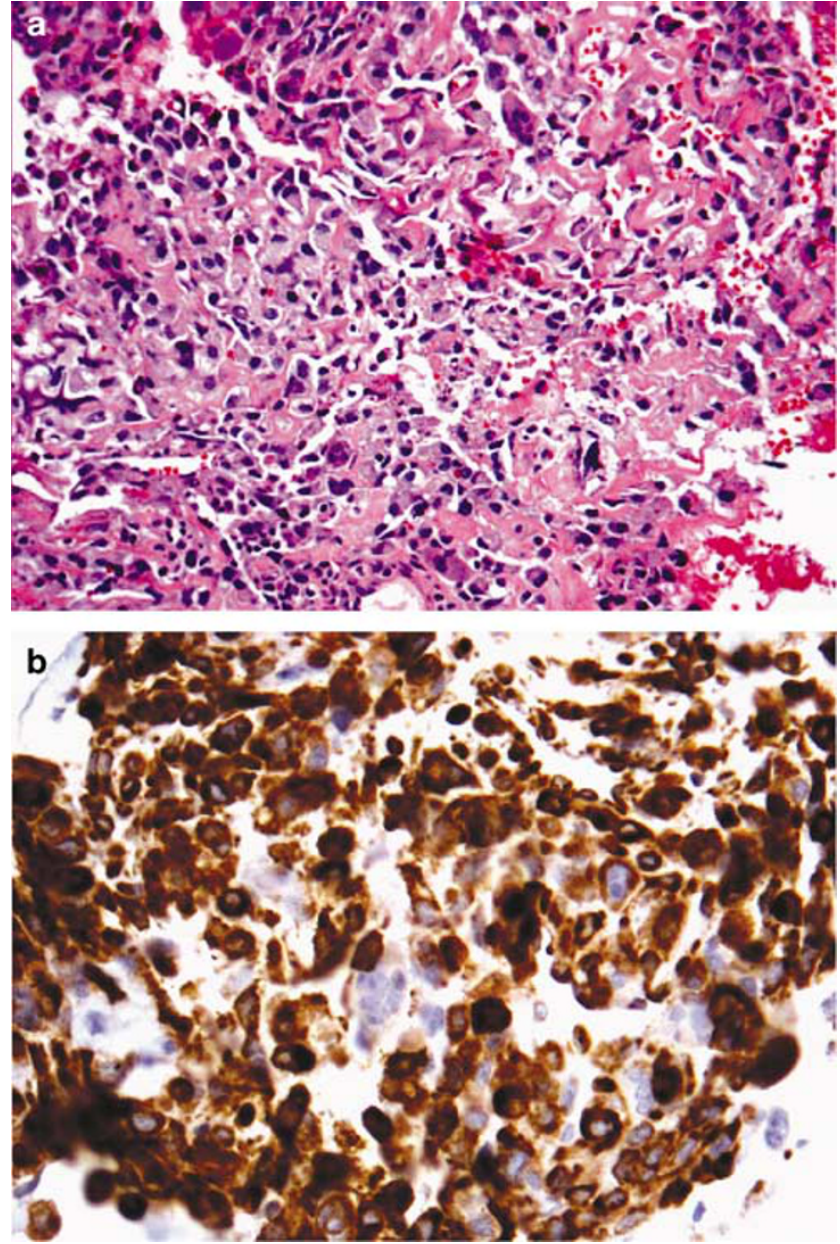

Figure 2 (a) Osteogenic sarcoma, high grade (case no. 14) hematoxylin and eosin $(\times 200)$. (b) Osteogenic sarcoma, high grade (case no. 14): strong and diffuse cytoplasmic staining for phospho-S6rp $(\times 400)$.

sarcoma, malignant solitary fibrous tumor and sarcoma NOS. In normal tissue around the tumors, we noted some staining of lymphocytes, fibroblasts and vascular smooth muscle.

\section{Discussion}

The serine/threonine kinase mTOR controls cell growth through several downstream effectors, including the p70S6K and the 4E-BP1 translational repressor, which regulate the S6rp and eIF-4E/G complex, respectively. ${ }^{7,15}$ Measurement of these downstream molecules by western blot, immunohistochemistry or other means could be useful to assess the activity of mTOR in tumors. Because p70S6K and 4E-BP1 are involved in the initiation of protein translation, inhibition of mTOR function by mTOR inhibitors abruptly inhibits the synthesis of critical proteins and profoundly blocks cell cycle progression at the G1 to S transition,,$^{9,11,12}$ and hence mTOR inhibition has potential therapeutic benefit.
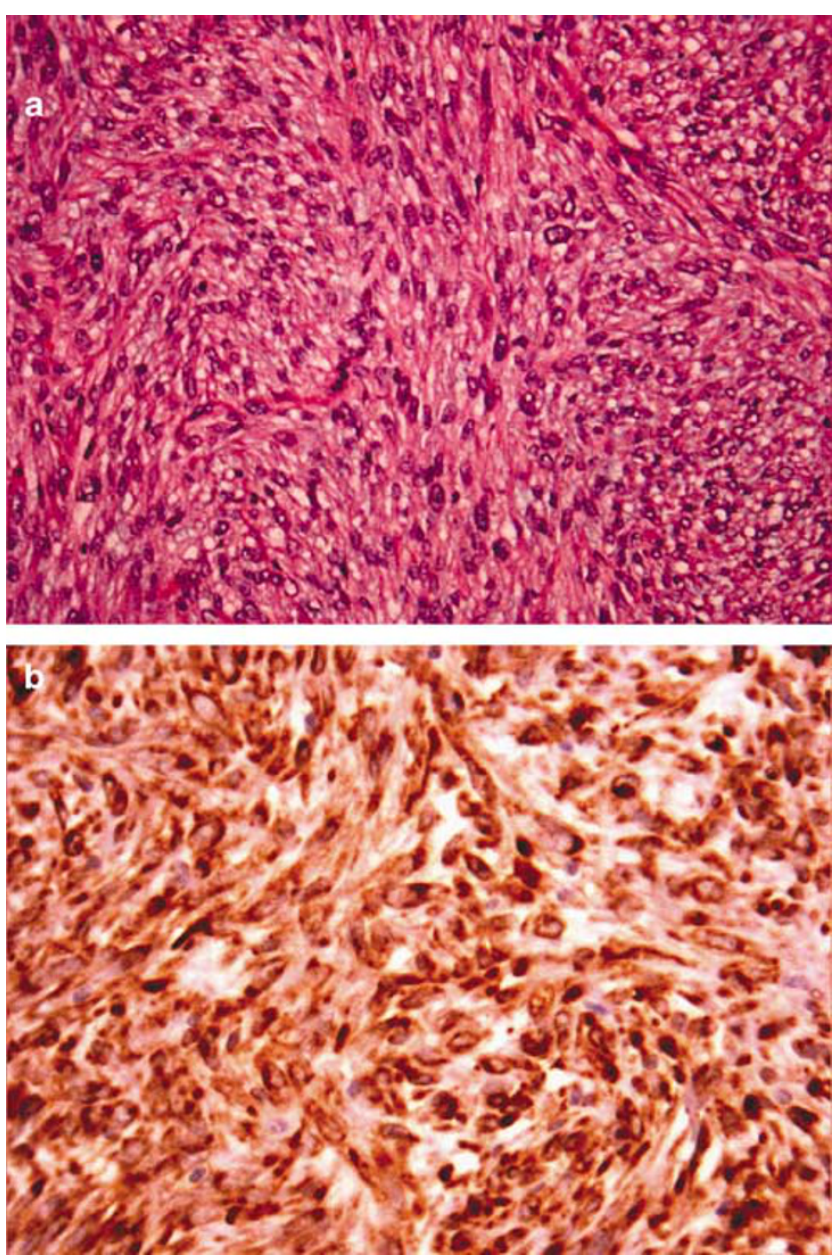

Figure 3 (a) Leiomyosarcoma (case no. 16): hematoxylin and eosin $(\times 200)$. (b) Leiomyosarcoma (case no. 16): strong and diffuse cytoplasmic staining for phospho-S6rp $(\times 400)$.

There are emerging data that the PI3K/AKT/ mTOR pathway is activated in a variety of solid organ and hematologic malignancies, including prostate carcinoma, malignant gliomas, endometrial carcinoma, melanoma, renal cell carcinoma, breast cancer, neuroblastoma, medulloblastoma, alveolar rhabdomyosarcoma, mantle cell lymphoma, and in post-transplant lymphoproliferative disorders. ${ }^{9,22,23}$ Therefore, screening of tumor specimens by IHC, FISH, gene sequencing or DNA microarray looking for PTEN loss, AKT activation, mTOR phosphorylation or any of its downstream moieties may provide a rational basis for identification of those cancer patients who might benefit from therapy with mTOR inhibitors. ${ }^{9}$

Our investigation is the first blinded analysis in which a downstream target of mTOR (phosphoS6rp) is correlated with early clinical response to an mTOR inhibitor (AP23573) administered alone or in combination to a cohort of different sarcoma patients. This study highlights the usefulness of phospho-specific antibodies to study cell signaling 
236
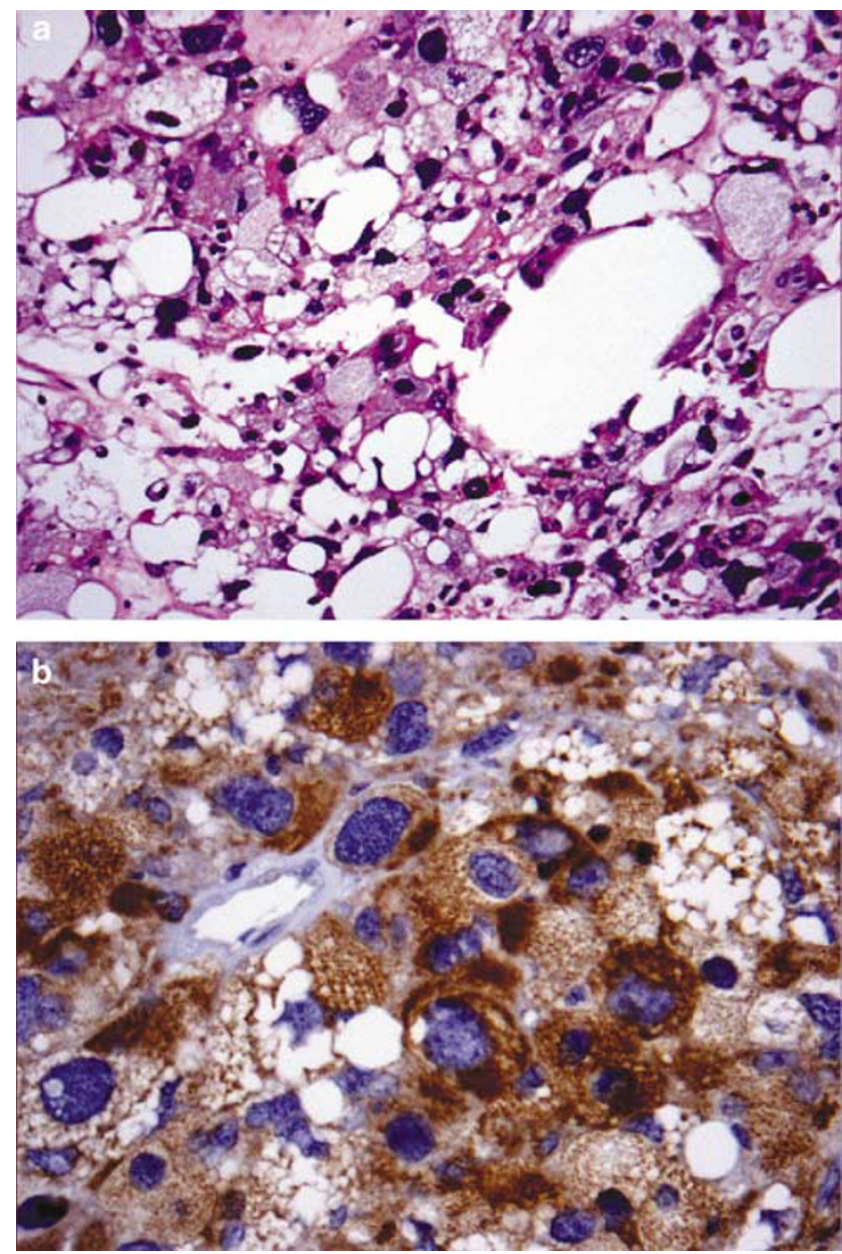

Figure 4 (a) Pleomorphic liposarcoma (case no. 19): hematoxylin and eosin $(\times 200)$. (b) Pleomorphic liposarcoma (case no. 19): strong and diffuse cytoplasmic staining for phospho-S6rp $(\times 400)$.

in patient-derived, paraffin-embedded tissues. The antibody used is directed against the defined phosphorylated serine or threonine moiety, which is a known step within the pathway under study and is critical for activation of the evaluated protein. Accordingly, selection of appropriate mTOR targets as biomarkers of activation of the mTOR pathway is of crucial importance. A previous study on constitutive activation of mTOR signaling pathway in post-transplant lymphoproliferative disorders ${ }^{22}$ found good correlation in the expression patterns of phospho-S6rp and phospho-4E-BP1. Immunohistochemical evaluation of p70S6K, although an attractive downstream protein of mTOR as a surrogate, could be misleading because of the structural similarity to p90S6K, which is not phosphorylated by the mTOR. ${ }^{23}$ Thus, we chose the phospho-S6rp as the mTOR surrogate for our study.

In our study, immunohistochemical reactivity was present in 15 of the 20 cases, with variation among cases, demonstrating that the phosphoS6rp antibody performed well in formalin-fixed, paraffin-embedded tissues. Further, the presence of variation within and across sarcoma histologic types signified that differences do exist among high-grade sarcomas with phospho-S6rp (mTOR pathway surrogate marker). This is similar to results found in ovarian cancer, in which differences in the expression of phospho-S6rp and other surrogate markers of mTOR (phospho-4E-BP1, p70S6K) were found in ovarian carcinomas and correlated in a multivariate analysis with high tumor grade and poor prognosis. ${ }^{24}$

Immunohistochemical quantitation of our cases nicely stratified into two groups, with a clear separation into low- and high-reactivity sets without knowledge of patient outcome. Of the $11 \mathrm{HE}, 8$ patients had stable disease $(73 \%)$ after 2 cycles of treatment with AP23573 alone or in combination with doxorubicin, and of the $9 \mathrm{LE}, 6$ patients had progressive disease $(67 \%)$, the differences between which were statistically significant. Thus, our data suggest that phospho-S6rp immunoreactivity could be predictive of early tumor response to AP23573.

We believe that our design, utilizing recurrent tumor tissue wherever possible (in $70 \%$ of cases) as opposed to the primary tumor, may be important, since it is possible that previous chemotherapy treatment may alter the activity of this and other pathways. Of note, within the LE group, there are three cases with none to low staining quantity that had stable disease. The reasons for this are not entirely clear but might be related to molecular events and/or delayed or suboptimal fixation.

Within the HE group, two of the three progressors had the lowest scores (20 and 25\%); accordingly, adjustment of the cutoff to $\leq 25 \%$ would lead to a high phospho-S6rp level that was strongly statistically significant for stable disease $(P \leq 0.005)$. The other patient in the HE group who had a high staining quantity but still had progressive disease might be explained by rapamycin/rapamycin analogue resistance. The mechanisms of resistance to rapamycin-based mTOR inhibitors are not entirely clear, but some investigators have implicated AKTmediated activation ${ }^{18}$ and expression of BCL-2. ${ }^{25,26}$ Others have suggested that the ability of rapamycin to inhibit c-myc induction correlates with intrinsic sensitivity, whereas the failure of rapamycin to inhibit induction or overexpression of c-myc correlates with resistance. ${ }^{27}$

In summary, our study suggests that the level of phospho-S6rp as judged by immunohistochemical staining is predictive of early tumor response to an mTOR inhibitor and that phospho-S6rp may be a promising new predictive sarcoma marker for targeted mTOR therapy. We also found that the mTOR pathway is variably activated in different sarcoma types and within specific sarcoma subsets.

Additional longer-term studies testing a large cohort in a prospective and independent manner are needed to confirm clinical utility. 


\section{References}

1 Longhi A, Errani C, De Paolis M, et al. Primary bone osteosarcoma: state of art. Cancer Treat Rev 2006;32: 423-436.

2 Tibes R, Trent J, Kurzrock R. Tyrosine kinase inhibitors and the dawn of molecular cancer therapeutics. Annu Rev Pharmacol Toxicol 2005;45:357-384.

3 Cassier PA, Dufresne A, Fayette J, et al. Emerging drugs for the treatment of soft tissue sarcomas. Expert Opin Emerg Drugs 2007;12:139-153.

4 Joensuu H, Roberts PJ, Sarlomo-Rikala M, et al. Effect of the tyrosine kinase inhibitor ST1571 in a patient with a metastatic gastrointestinal stromal tumor. N Engl J Med 2001;344:1052-1056.

5 Rubin BP, Schuetze SM, Eary JF, et al. Molecular targeting of platelet-derived growth factor B by imatinib mesylate in a patient with metastatic dermatofibrosarcoma protuberans. J Clin Oncol 2002;20:3586-3591.

6 Dufner A, Andjelkovic M, Burgering BM, et al. Protein kinase B localization and activation differentially affect S6 kinase 1 activity and eukaryotic translation initiation factor 4E-binding protein 1 phosphorylation. Mol Cell Bio 1999;19:4525-4534.

7 Tee AR, Blenis J. mTOR, translational control and human disease. Semin Cell Dev Biol 2004;16:29-37.

8 Parsons R. Human cancer, PTEN and the PI-3 kinase pathway. Semin Cell Dev Biol 2004;15:171-176.

9 Vignot S, Faivre S, Aguirre D, et al. mTOR-targeted therapy of cancer with rapamycin derivatives. Ann Oncol 2005;16:525-537.

10 Dennis PB, Fumagalli S, Thomas J. Target of rapamycin (TOR): balancing the opposing forces of protein synthesis and degradation. Curr Opin Genet Dev 1999;9:49-54.

11 Bjornsti M, Houghton PJ. The TOR pathway: a target for cancer therapy. Nat Rev Cancer 2004;4:335-348.

12 Rowinsky EK. Targeting the molecular target of rapamycin (mTOR). Curr Opin Oncol 2004;16:564-575.

13 Dufner A, Thomas J. Ribosomal S6 kinase signaling and the control of translation. Exp Cell Res 1999;253:100-109.

14 Ferrari S, Bandi HR, Hofsteenge J, et al. Mitogenactivated $70 \mathrm{~K}$ S6 kinase. Identification of in vitro $40 \mathrm{~S}$ ribosomal S6 phosphorylation sites. J Biol Chem 1991; 266:22770-22775.

15 Sarbassov DD, Sabatini DM. Redox regulation of the nutrient-sensitive raptor-mTOR pathway and complex. J Biol Chem 2005;280:39505-39509.
16 Peng T, Golub TR, Sabatini DM. The immunosuppressant rapamycin mimics a starvation-like signal distinct from amino acid and glucose deprivation. Mol Cell Biol 2002;22:5575-5584.

17 Takano A, Usui I, Haruta T, et al. Mammalian target of rapamycin pathway regulates insulin signaling via subcellular redistribution of insulin receptor substrate 1 and integrates nutritional signals and metabolic signals of insulin. Mol Cell Biol 2001;21: 5050-5062.

18 Petroulakis E, Mamane Y, Bacquer OL, et al. mTOR: implications for cancer and anticancer therapy. $\mathrm{Br}$ J Cancer 2006;94:195-199.

19 Chawla SP, Tolcher AP, Staddon AP, et al. Survival results with AP23573, a novel mTOR inhibitor, in patients with advanced soft tissue or bone sarcomas: update of phase II trial. J Clin Oncol 2007;25(June 20 Suppl):10076.

20 Therasse P, Arbuck SG, Eisenhauer EA, et al. New guidelines to evaluate the response to treatment in solid tumors. J Natl Cancer Inst 2000;92:205-216.

21 Coindre JM, Terrier P, Guillou L, et al. Reproducibility of a histopathologic grading system for adult soft tissue sarcoma. Cancer 1986;58:306-309.

22 Vega F, Medeiros LJ, Atwell C, et al. Activation of mammalian target of rapamycin signaling pathway tumor cell survival in anaplastic lymphoma kinasepositive anaplastic large cell lymphoma. Cancer Res 2006;66:6589-6597.

23 El-Salem M, Raghunath PN, Marzec M, et al. Constitutive activation of mTOR signaling pathway in posttransplant lymphoproliferative disorders. Lab Invest 2007;87:29-39.

24 Castellvi J, Garcia A, Rojo F, et al. Phosphorylated 4E binding protein 1: a hallmark of cell signaling that correlates with survival in ovarian cancer. Cancer 2006;107:1801-1811.

25 Aguirre D, Boya P, Bellet D, et al. Bcl-2 and CCND1/ CDK4 as molecular markers of the cellular effects of mTOR inhibitors in human ovarian carcinoma cells. Apoptosis 2004;9:797-805.

26 Dilling MB, Germain GS, Dudkin L, et al. 4E-binding proteins, the suppressors of eukaryotic initiation factor $4 \mathrm{E}$, are down-regulated in cells with acquired or intrinsic resistance to rapamycin. J Biol Chem 2002;277:13907-13917.

27 Hosoi H, Dilling MB, Liu LN, et al. Studies on the mechanism of resistance to rapamycin in human cancer cells. Mol Pharmacol 1998;54:815-824. 